\title{
Autologous Anti-CD19 Chimeric Antigen Receptor T-cells C-CAR011
}

National Cancer Institute

\section{Source}

National Cancer Institute. Autologous Anti-CD19 Chimeric Antigen Receptor T-cells C-

CAR011. NCI Thesaurus. Code C141050.

A proprietary preparation of autologous T-lymphocytes that have been genetically modified and transduced with a lentiviral vector expressing a second-generation chimeric antigen receptor (CAR) targeting the tumor-associated antigen (TAA) CD19 and containing, as of yet undisclosed, costimulatory signaling domains, with potential immunostimulating and antineoplastic activities. Upon administration, autologous antiCD19 CAR T-cells C-CAR011 target and bind to CD19-expressing tumor cells, thereby inducing selective toxicity in CD19-expressing tumor cells. CD19 antigen is a B-cell specific cell surface antigen expressed in all B-cell lineage malignancies. 